\title{
Earth's pole coordinates determined from Lageos-1/2 laser ranging
}

\author{
V.P. Zhaborovskyy ${ }^{1 *}$ V. Ya. Choliy ${ }^{1,2}$ \\ ${ }^{1}$ Main Astronomical Observatory of NAS of Ukraine, Akademika Zabolotnoho str., 27, 03680 Kyiv, Ukraine \\ ${ }^{2}$ Taras Shevchenko National University of Kyiv, Glushkova ave., 4, 03127, Kyiv, Ukraine
}

\begin{abstract}
The Earth's pole coordinates, obtained from satellite laser ranging data of Lageos- 1 and Lageos- 2 are presented. The procedure, used models and algorithms are described. Results are compared with similar series from IERS database.
\end{abstract}

Key words: methods: data analysis, reference system, geodesy

\section{INTRODUCTION}

During the past 40 years the precision of Satellite Laser Ranging (SLR) has changed dramatically. Together with greater computational capabilities and better algorithms for data storage, searching, and analysis, the overall precision of the geodynamics and geodetic tasks was substantially improved. Among the methods substantially improved, the determination of the Earth' pole motion and irregularities of Earth' spin velocity are the central ones. The Lageos-1 (1976) and Lageos-2 (1992) satellites were launched specifically to accomplish these tasks. These satellites are modelled as mathematical pointmass. They are spherically shaped, and their orbits were designed to diminish the influence of different poorly-modelled accelerations, as to allow relatively effortless laser ranging. The processing of the observations allows the International Earth Rotation Service to create and distribute official time corrections and pole coordinate series for users worldwide. Such a result is not possible without extensive international collaboration and independent data analysis centres capable of creating independent solutions. These are the essential components of the work coordinated by IERS.

Since 1987, a data analysis centre of satellite laser ranging (SLR) has been in operation on a permanent basis at the Main Astronomical Observatory of NAS . The previously used software package KyivGeodynamics is outdated, as over the past ten years the IERS introduced significant changes to their Standards [5] three times. That is why the new version of the code was created [8], called KyivGeodynamics++ (hereinafter $\mathrm{KG++}$ ). To validate the new code the processing of the SLR data for 2001

\footnotetext{
*zhskyy@gmail.com

(C) V.P. Zhaborovskyy, V. Ya. Choliy, 2014
}

was conducted.

\section{THE PROCEDURE OF CALCULATION}

The algorithm of data processing is based upon the linearised Least Squares method. The observed distance between the satellite and the station $\vec{\rho}$ can be written as:

$$
\vec{\rho}(t)=\hat{\mathbb{M}}(t) \vec{r}(t)-\vec{R}(t)=\vec{\Phi}(\vec{X}, t),
$$

where $\vec{r}(t)$ is satellite position in International Celestial Reference Frame (ICRF), $\vec{R}$ is the station position in the International Terrestrial Reference Frame (ITRF), and $\mathbb{M}$ is the transformation matrix between ITRF and ICRF. Another vector $\vec{\Phi}(\vec{X}, t)$ is the vector function (in general case it is highly complicated with regards to the calculation) of the parameters $\vec{X}$.

Observation instruments (telescope, equipped with laser transmitter and receiver) allow us to observe the absolute value of, say $\vec{\rho}$, the distance to the satellite. Having approximate values for $\vec{X}_{0}$ the (1) can be linearised, and then

$$
\rho_{o}-\rho_{c}=\left.\left(\frac{\partial \vec{\Phi}(\vec{X})}{\partial \vec{X}}\right)\right|_{\vec{X}_{0}} \vec{x},
$$

where $\vec{x}=\vec{X}-\vec{X}_{0}$ are corrections to the parameters. A sufficient number of observations will give us the possibility to determine a better estimation for $\vec{X}$.

The observational data is processed using a twostage procedure. During the first stage the Kepler 
orbital elements of the satellite are estimated. This step is repeated until the standard deviation of the difference observed minus calculated stops decreasing. During the second stage, the determined orbit is used to estimate the pole coordinates along with empirical acceleration of the satellite. The latter acceleration is widely used to compensate any forgotten accelerations of different origin not explicitly introduced in the satellite equation of motion.

The elementary processing unit is the arc. It comprises the observation of a single satellite on a 7-day interval (standard IERS value) together with a full set of vector $\vec{X}$ components derived from it.

All of the observations of Lageos- 1 and Lageos- 2 satellites for the year 2001 were processed for this article. There were approximately 75000 normal points in our database. There were 52 seven-day-long arcs: a end point of an arc served as the starting point for the next one.

Each arc was processed in two stages. During the first stage only Keplerian orbital elements were estimated until the overall standard deviation of the observed minus calculated values stopped decreasing. This stage lasted 4-5 iterations. Following this, the estimation of the empirical acceleration (one value per day) and pole coordinates (two values per day) took place. Iterations continued until standard the deviation decreased to below $10 \mathrm{~cm}$. The specified $10 \mathrm{~cm}$ value is sufficient to estimate pole coordinates. In general, the overall process took $12-15$ iterations per arc. The observed minus calculated residuals were thoroughly analysed during the iterations, and their distribution was checked for normality. Rejection criteria was set to $3 \sigma$. Sometimes up to $30 \%$ of the observations were dropped.

New code strictly follows all recommendations from IERS Conventions 2010 [5]. All software modules were written from scratch and tested separately on artificial examples before being introduced into productive code. Processing of the Lageos- 1 and Lageos- 2 data is the first result from the release version code.

A brief outline of the models used is presented below. If not specified, the models from IERS Conventions were used.

FORCES:

- Earth's gravity field and ocean tide model EGM96 [6], $20 \times 20$ solution;

- Third body gravity: Moon, Sun and all planets - DE421 [4];

- Direct solar radiation pressure: reflectance coefficient a priori 1.14;

- Indirect light pressure: using cylindrical shadow function;
- Relativistic correction.

REFERENCE FRAME:

- Stations coordinates and stations velocities: ITRF2005 solution, epoch 2000.0 [1];

- Precession and nutation: full IAU2000A model [2];

- Polar motion: C04 IERS;

- Tidal effects.

OTHER:

- Observations: all normal points for 2001 year from CDDIS database for Lageos-1 and Lageos2 ;

- Tropospheric refraction: Marini/Murray model [7];

- Constants (speed of light, gravitational constant etc.) from IERS Conventions.

ESTIMATED PARAMETERS:

- Estimation criteria: $3 \sigma \simeq 5 \mathrm{~cm}$ for final result;

- O-C filter: $3 \sigma$ for each iteration;

- Satellite orbital elements are determined as one set per 7 days arc;

- Pole coordinates determined for each day;

- Empirical acceleration coefficient determined for each day.

\section{RESULTS}

The $\mathrm{X}$ and $\mathrm{Y}$ pole coordinates were determined for every day of 2001 from the observations of a single satellite. The results are presented in Fig. 1 and Fig. 2 for Lageos-1 and Lageos-2. The standard deviation for the last iteration of each one-day arc does not exceed $10 \mathrm{~cm}$ for Lageos- 1 and $7 \mathrm{~cm}$ for Lageos- 2 .

This result was compared with analogous data obtained in different international data analysis centres. All of these data are available online ${ }^{1}$. We used the series by the Center for Space Research, USA (crs), Delft University of Technology, Netherland (dut), Institute for Applied Astronomy, Russia (iaa) and standard ILRS solutions (ilrs) (see Fig. 3).

The method of comparison is based upon Helmert transform and described in [3]. On the first stage, having two different sets of polar data, one can extract systematic differences of these sets by applying a 6-parameter Helmert transform:

\footnotetext{
${ }^{1}$ ftp://hpiers.obspm.fr/iers/series/operational
} 


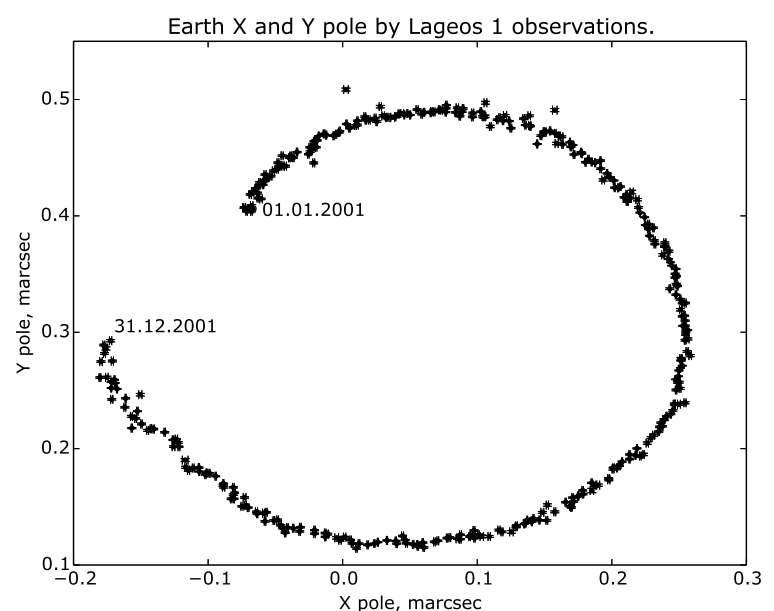

Fig. 1: Earth pole from Lageos-1 observation.

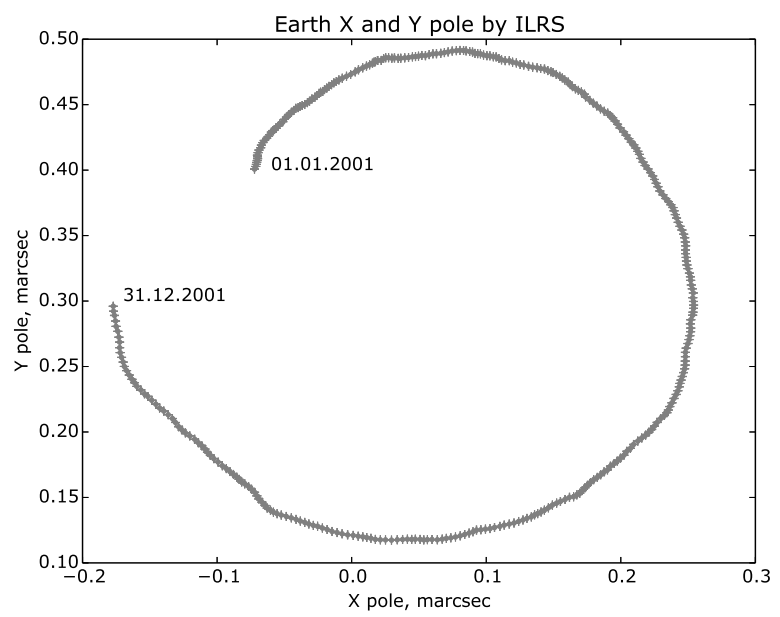

Fig. 3: Earth pole from ILRS.

$$
\vec{r}_{2}-\vec{r}_{1}=\left[\begin{array}{ll}
a_{11} & a_{12} \\
a_{21} & a_{22}
\end{array}\right] \vec{r}_{1}+\left[\begin{array}{l}
b_{1} \\
b_{2}
\end{array}\right],
$$

where $\vec{r}$ is the vector containing two pole coordinates, where systematics error are described as rotations $\left(a_{i j}, i \neq j\right)$ scales $\left(a_{i i}\right)$ and shifts $\left(b_{k}\right)$. Following this, mutual standard deviations $\sigma_{i j}$ of series are obtained:

$$
\sigma_{i j}^{2}=\frac{\sum_{k=1}^{N}\left(\vec{r}_{(i) k}-\vec{r}_{(j) k}^{*}\right)^{2}}{N-M},
$$

where $M$ is the number of degrees of freedom, and the symbol $*$ is used to signal the series without systematic errors. The own standard deviations of the series may be obtained if a sufficient number of $\sigma_{i j}$

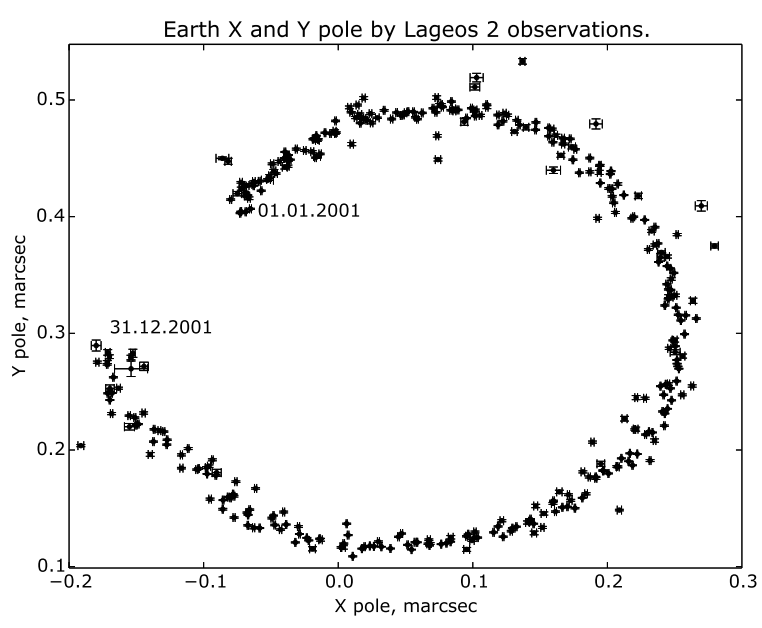

Fig. 2: Earth pole from Lageos-2 observation.

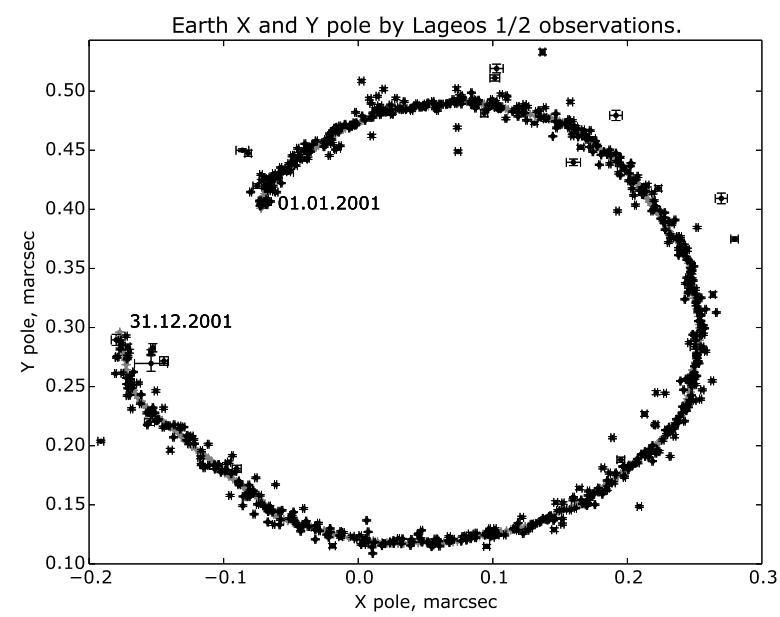

Fig. 4: Earth pole - all results.

is obtained from the equation:

$$
\left\{\begin{array}{l}
\sigma_{12}^{2}=\sigma_{1}^{2}+\sigma_{2}^{2}-2 \rho_{12} \sigma_{1} \sigma_{2} \\
\sigma_{13}^{2}=\sigma_{1}^{2}+\sigma_{3}^{2}-2 \rho_{13} \sigma_{1} \sigma_{3} \\
\sigma_{23}^{2}=\sigma_{2}^{2}+\sigma_{3}^{2}-2 \rho_{23} \sigma_{2} \sigma_{3}
\end{array}\right.
$$

where $\rho_{i j}$ are the correlations of the corresponding series.

Varying solutions were used as a base for comparison. Three series were compared. The computed standard deviations are given in Table 1 where every cell contains the resulting value found by comparing our satellite solution (row name) and two centre names given as the column headings. The mean standard deviation of pole coordinates obtained from Lageos- 1 observations is $3.10 \times 10^{-4}$, and that obtained from Lageos-2 observations is $1.19 \times 10^{-3}$.

All series of pole coordinates are present in Fig. 4. 
Table 1: Standard deviations in mas of some Earth pole coordinates series.

\begin{tabular}{cccccc}
\hline & dut - csr & csr - iaa & iaa - ilrs & ilrs - dut & mean \\
\hline Lag1 & $2.98 \cdot 10^{-4}$ & $3.57 \cdot 10^{-4}$ & $2.44 \cdot 10^{-4}$ & $3.42 \cdot 10^{-4}$ & $3.10 \cdot 10^{-4}$ \\
Lag2 & $1.27 \cdot 10^{-3}$ & $1.22 \cdot 10^{-3}$ & $1.21 \cdot 10^{-3}$ & $1.04 \cdot 10^{-3}$ & $1.19 \cdot 10^{-3}$ \\
\hline
\end{tabular}

\section{CONCLUSIONS}

The values presented in Table 1 show two main points. Firstly, the precision of Lageos-1 based pole coordinates are nearly one order of magnitude better than the values from processing Lageos-2 data. The second point is that in any case both of the precisions agree with values produced by other data analysis centres. This result demonstrates the correct work of $\mathrm{KG}++$ on $2 \mathrm{~cm}$ precision level and its applicability for the analysis of the SLR data with precision sufficient for IERS tasks.

\section{REFERENCES}

[1] Altamimi Z., Collilieux X., Legrand J., Garayt B. \& Boucher C. 2007, J. Geophys. Res., 112, B09401
[2] Capitaine N., Chapront J., Lambert S. \& Wallace P. T. 2003, A\&A, 400, 1145

[3] Choliy V. Ya. 2014, Advances in Astronomy and Space Physics, 4, 15

[4] Folkner W. M., Williams J. G. \& Boggs D. H. 2009, The Interplanetary Network Progress Report, 178, 1

[5] IERS Technical Note No. 36 (IERS Conventions 2010), 2010, IERS Conventions Center

[6] Lemoine F., Kenyon S., Factor J. et al. 1998, 'The Development of the Joint NASA GSFC and National Imagery and Mapping Agency (NIMA) Geopotential Model EGM96', NASA/TP-1998-206861, Goddard Space Flight Center, Greenbelt

[7] Marini J. \& Murray C. 1973, 'Correction of laser range tracking data for atmospheric refraction at elevations above 10 degrees', NASA-TM-X-70555, GSFC, Greenbelt

[8] Choliy V. Ya. \& Zhaborovskyy V. P. 2011, Advances in Astronomy and Space Physics, 1, 96 
Advances in Astronomy and Space Physics 